
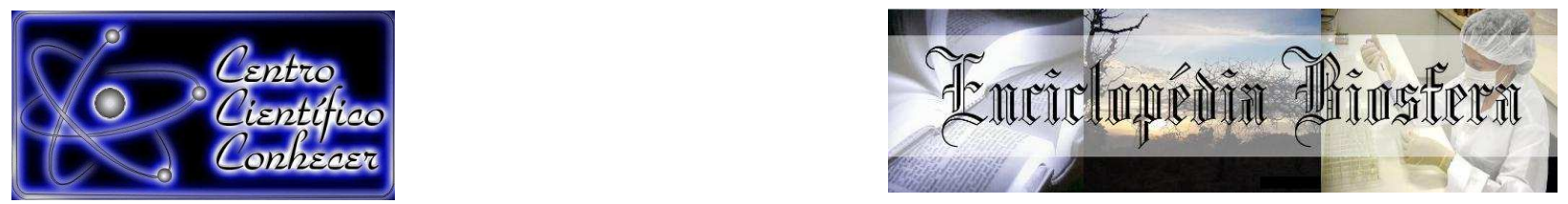

\title{
O OLHAR DA CIÊNCIA PARA A MITIGAÇÃO DAS SECAS
}

\section{Renata Firmino do Amaral'1, Patrícia Verônica Pinheiro Sales Lima², Francisco Elicivaldo Lima ${ }^{3}$}

1 Doutoranda do Programa de Pós-Graduação em Desenvolvimento e Meio Ambiente da Universidade Federal do Ceará (amaralrenataf@gmail.com) Fortaleza- Brasil

2 Profa Dra do Departamento de Economia Agrícola da Universidade Federal do Ceará , Fortaleza- Brasil

3 Prof. Dr. do Departamento de Engenharia Mecânica da Universidade Federal do Ceará , Fortaleza- Brasil

Recebido em: 02/10/2017 - Aprovado em: 21/11/2017 - Publicado em: 05/12/2017 DOI: 10.18677/EnciBio_2017B104

\begin{abstract}
RESUMO
Este estudo buscou mapear a literatura científica relevante sobre mitigação das secas, a partir de indicadores bibliométricos. Para tanto, foi realizada busca dos termos "drought" e "mitigation" na base de dados Web of Science. O período de análise abrangeu os anos compreendidos entre 1970 a 2016. Foram selecionados 134 documentos, demonstrando que os estudos sobre mitigação dos impactos das secas são relativamente reduzidos frente às publicações sobre mudanças climáticas. No entanto, houve um crescimento significativo no número de documentos publicados no período analisado. Foi possível perceber que a mitigação das secas é abordada sob três perspectivas principais de pesquisa: "mudanças climáticas", "impactos das secas" e "gestão e modelagem climática". Os países que mais produziram trabalhos na área foram Estados Unidos, China e Índia. O Brasil contribuiu com 2,2\% do total de publicações. Apesar de se tratar de um tema de abrangência mundial, observou-se que a colaboração entre os autores se dá de forma tímida, o que pode inibir avanços qualitativos nessa temática, seja em escala global ou regional.
\end{abstract}

PALAVRAS-CHAVE: Bibliometria. Pesquisa em mitigação. Redes de cooperação

\section{THE LOOK OF SCIENCE FOR DROUGHT MITIGATION}

\begin{abstract}
This study was aimed to map the relevant scientific literature on mitigating the effects of drought, based on bibliometric indicators. For this purpose, the terms "drought" and "mitigation" were searched, in the Web of Science database. The analysis covered the period from 1970 to 2016. 134 documents were selected, showing that studies on mitigating the effects of droughtare relatively few when compared with the publications on climate change. However, there was a significant increase in the number of documents published during the analyzed period. It was possible to understand that drought mitigation is discussed from three perspectives of research: "climate change", "drought impacts" and "climate modeling and management". The countries which produced the most work in the discipline were the United States, China and India. Brazil contributed with $2.2 \%$ of the total publications.Although it is a worldwide subject, it was noted that the collaboration between authors occurs timidly, which may lead to inhibit qualitative advances in this field, on a regional or global scale.
\end{abstract}

KEYWORDS:Bibliometrics. Research on mitigation.Cooperation networks ENCICLOPÉDIA BIOSFERA, Centro Científico Conhecer - Goiânia, v.14 n.26; p.1239 


\section{INTRODUÇÃO}

Nos últimos anos os períodos de estiagem estão se tornando cada vez mais freqüentes e intensos (MANCAL et al., 2016), o que corrobora as projeções do Intergovernmental Panel on Climate Change que apontam para a intensificação das secas em escala global, especialmente nas áreas áridas e semiáridas (IPCC, 2014). O cenário observado tem atraído a atenção de governos, agências de desenvolvimento e de pesquisadores das mais diferentes áreas no sentido de elaborar estratégias capazes de mitigar os impactos das secas. No entanto, apesar dos esforços despendidos as secas ainda causam prejuízos substanciais às populações atingidas. Nota-se a necessidade de pesquisas que levem a inovações capazes de minimizar os danos causados pela seca tanto no curto quanto no longo prazo. Miyan (2015), afirma que as populações atingidas pelo fenômeno devem buscar estratégias para mitigação dos seus efeitos.

As pesquisas sobre secas consistem em um desafio para os cientistas elucidarem (VAN LOON, 2015). Isso porque embora existam estudos sendo desenvolvidos por pesquisadores de diferentes partes do mundo percebe-se que a maioria volta-se para modelos de previsão de curto prazo e distribuição espacial das secas a partir de análises pontuais feitas em locais específicos ou pequenas regiões (VAN LOON, 2015).

A sistematização de informações sobre a evolução da produção científica voltada para a mitigação dos impactos das secas favorece a atuação de governos e agências no planejamento de medidas mais efetivas e ajude a identificar novos tópicos a serem estudados dentro dessa linha de pesquisa. Acrescente-se, ainda, que a divulgação de estudos é importante para a comunidade científica, mas também para a sociedade civil que utiliza o conhecimento produzido para alcançar o desenvolvimento social (SILVEIRA, 2012).

Sob essa perspectiva, este artigo teve como objetivo analisar a produção do conhecimento sobre "mitigação da seca", identificando a evolução das publicações científicas por meio de uma análise bibliométrica de artigos fontes. Apesar da crescente aplicação da bibliometria nos diferentes campos de pesquisa, ainda não existem documentos sobre esse tema. Os estudos mais relacionados foram encontrados na temática "mudanças climáticas" e não abordam a problemática da seca de forma específica. É o caso das publicações de Ji et al. (2014), Wang et al. (2014), Marx et al.(2016) e Haunschild et al.(2016).

\section{MATERIAL E MÉTODOS}

O método adotado no estudo foi a análise bibliométrica, a qual consiste em uma técnica estatística que utiliza indicadores para medir a produção científica e acompanhar a evolução de diferentes áreas da ciência para auxiliar a gestão e tomada de decisão da pesquisa (COLEPÍCOLO, 2014). Os procedimentos adotados constaram de quatro etapas, conforme recomendação de Wang et al. (2014), e são descritos a seguir. A pesquisa foi realizada no mês de outubro de 2016.

i) ETAPA 1- O ponto de partida da análise bibliométrica foi a definição dos termos de pesquisa: "mitigation" e "drought". Os termos foram incluídos entre aspas para garantir maior precisão nos registros obtidos. Além disso, foi utilizado o operador "and".

A busca aos termos foi feita por meio de consulta eletrônica à base Web of Science (WoS). A escolha dessa base é justificada pela abrangência mundial (mais de 80.000 livros acadêmicos, 18.000 periódicos científicos e 180.000 atas da 
conferência segundo o website Clarivate Analytics) e maior aceitação no âmbito internacional (WANG et al., 2014). Embora não seja completa, permite acessar a maior parte dos estudos relevantes sobre questões climáticas em escala mundial. 0 período pesquisado abrangeu os anos de 1970 a 2016. Nessa fase foram capturados 1.106 documentos;

ii) Etapa 2 - Após uma seleção inicial foi realizado um refinamento porque percebeu-se que muitos textos continham as palavras da busca, porém não estavam "afinados" com o tema de pesquisa, isto é, tratavam de assuntos não relacionados diretamente com mitigação das secas. Ainda nessa fase optou-se por excluir os documentos que não foram citados em outros trabalhos, por inferir que os mesmos possuem baixo reconhecimento científico. No entanto, permaneceram os textos publicados nos anos de 2014, 2015 e 2016, admitindo-se que possuiam potencial para reconhecimento científico futuro (ENSSLIN et al., 2012). Após essa filtragem restaram 134 textos;

iii) Etapa 3 - Essa etapa consistiu na adição do termo "semiarid" aos dois anteriores e repetição dos procedimentos descritos nas duas etapas iniciais. Esse novo termo permitiu capturar 18 textos, restando 10 após o refinamento;

iv) Etapa 4 - A última fase consistiu na obtenção dos indicadores bibliométricos. Para tanto foram utilizadas ferramentas disponibilizadas no site do WoS e no software VOSViewer. Os indicadores bibliométricos avaliados foram: a) número de publicações (para avaliar a tendência de crescimento), b) palavras-chave (para identificar os tópicos mais abordados, c) origem das publicações (principais países) e d) autores chave e redes de cooperação.

\section{RESULTADOS E DISCUSSÃO}

Os resultados discutidos a seguir sintetizam o estudo bibliométrico sobre mitigação das secas a partir de um conjunto representativo de documentos científicos sobre o tema. Na sequência são apresentados: i) o crescimento da produção mundial de publicação sobre a temática,ii) os tópicos mais abordados, iii) a distribuição espacial das pesquisas e iv) os principais pesquisadores e as redes de cooperação.

\section{A Evolução das Pesquisas}

A pesquisa realizada inicialmente com as palavras-chave drought e mitigation resultou, após o refinamento dos textos, em 134 documentos/publicações versando entre artigos em periódicos, revisões, reuniões científicas (congressos, seminários, workshops), editoriais e patentes, sendo que $82 \%$ dos textos são artigos. Quanto às reuniões, algumas destas são bem específicas para a mitigação da seca, dentre estas estão o Workshop sobre Apoio à Decisão de um Sistema de Mitigação de Impactos da Seca nas regiões mediterrâneas (Workshop on a Decision Support System for Mitigation of Drought Impacts in the Mediterranean Regions). Como o próprio título da conferência sugere, no evento foram discutidas tecnologias para a mitigação da seca na região do Mediterrâneo.

Os trabalhos apresentados no workshop demonstram a preocupação Comissão Europeia, juntamente com a cooperação internacional com países mediterrâneos, com a questão da seca nesta região. É o caso do estudo de Rossi; Cacelliere (2003) que apresenta um pacote de software para identificar e avaliar a seca, em nível local, e assim auxiliar os tomadores de decisão na gestão dos recursos hídricos em períodos de seca. $\mathrm{O}$ evento reforçou que, para esses países, o principal problema acarretado pela seca é a escassez de água e esta por sua vez 
traz prejuízos ambientais e às atividades humanas (DZIEGIELEWSKI, 2003). Dentre as atividades que estão aumentando a demanda por água nesta região está o turismo e a agricultura (ROSSI; CACELLIERE, 2003).

No quadro 1 estão apresentados outros encontros que tratam diretamente ou indiretamente da questão da mitigação da seca. Nota-se, portanto, a preocupação mundial com o tema e a ocorrência de reuniões que possibilitam a integração entre os agentes envolvidos com a seca em diferentes partes do mundo.

QUADRO 1 Publicações em conferências envolvendo o tema mitigação da seca

\begin{tabular}{|c|c|c|}
\hline Título da conferência & $\begin{array}{l}\text { Número } \\
\text { de } \\
\text { trabalhos }\end{array}$ & $\begin{array}{l}\text { Ano da } \\
\text { conferência }\end{array}$ \\
\hline $\begin{array}{l}\text { International Workshop on Agrometeorology in the 21st Century - } \\
\text { Needs and Perspective }\end{array}$ & 1 & 1999 \\
\hline $\begin{array}{c}\text { Export-Group Meeting on Early Warning Systems for Drought } \\
\text { Preparedness and Drought Management }\end{array}$ & 1 & 2000 \\
\hline $\begin{array}{c}\text { Workshop on a Decision Support System for Mitigation of } \\
\text { Drought Impacts in the Mediterranean Regions }\end{array}$ & 3 & 2001 \\
\hline $\begin{array}{c}\text { European Conference on Desalination and the Environment Water } \\
\text { Shortage } \\
\text { 3rd International Symposium on Alternative Ways to Combat }\end{array}$ & 2 & 2001 \\
\hline $\begin{array}{c}\text { Desertification, Connecting Community Action with Science and } \\
\text { Common Sense }\end{array}$ & 1 & 2002 \\
\hline Meeting on Natural Disasters and Extreme Events in Agriculture & 2 & 2004 \\
\hline $\begin{array}{l}\text { Symposium on the Impact of Climate on Groundwater Resources } \\
\text { held at the 32nd International Geological Congress }\end{array}$ & 1 & 2004 \\
\hline EGU General Assembly on Integrated Water Resource Assessment & 1 & 2004 \\
\hline $\begin{array}{l}\text { 26th Meeting of the International-Association-of-Agricultural- } \\
\text { Economists (IAAE) }\end{array}$ & 1 & 2006 \\
\hline 5th Study Conference on BALTEX & 1 & 2007 \\
\hline 8th waternet/Warfsa/GWP SA Symposium & 5 & 2007 \\
\hline $\begin{array}{c}\text { 6th World FRIEND Conference on Global Change: Facing Risks and } \\
\text { Threats to Water Resources }\end{array}$ & 1 & 2010 \\
\hline $\begin{array}{l}\text { International Conference on Modern Hydraulic Engineering (CMHE) } \\
\text { Conference on Transforming Rural Livelihoods in Africa - How Can }\end{array}$ & 1 & 2012 \\
\hline $\begin{array}{l}\text { Land and Water Management Contribute to Enhanced Food Security } \\
\text { and Address Climate Change Adaptation and Mitigation }\end{array}$ & 1 & 2013 \\
\hline 21st International phd Students Conference & 1 & 2014 \\
\hline $\begin{array}{l}\text { 14th InternationalMultidisciplinaryScientificGeoconference (SGEM) } \\
\text { 3rd Remote Sensing and Hydrology Symposium (RSHS 14) / 3rd }\end{array}$ & 1 & 2014 \\
\hline $\begin{array}{c}\text { International Conference of GIS/RS in Hydrology, Water Resources } \\
\text { and Environment (ICGRHWE 14) }\end{array}$ & 2 & 2014 \\
\hline Joint Inter-Association Symposium on Extreme Hydrological Events & 2 & 2015 \\
\hline
\end{tabular}

Fonte: Elaboração própria de acordo com dados obtidos no site Web of Science

Duas conferencias tratam diretamente da questão da seca como o já citado Workshop on a DecisionSupport System for Mitigation of Drought Impacts in the Mediterranean Regions e a Export-Group Meetingon Early Warning Systems for Drought Preparedness and Drought Management, sendo que esta segunda trata da mitigação da seca a partir da prevenção de períodos de seca e na gestão do problema se caso a seca ocorra. As outras conferências tratam da questão da gestão da água, do meio ambiente da agricultura, ou seja, de temas que estão ligados indiretamente à seca.

Segundo Haunschild et al., (2016) o número de publicações é a medida mais utilizada para quantificar a produção científica e o número de citações é o indicador mais frequente para avaliar a qualidade de tal produção. Com relação à evolução 
dos trabalhos relacionados ao tema em questão, pode-se observar na Figura 1a, que desde o ano 2000 está havendo um aumento significativo nas publicações. Essa tendência crescente na literatura sobre mitigação das secas acompanha o comportamento exponencial observado nas publicações sobre mudanças climáticas destacadas por Bornmann; Mutz (2015). Para Haunschild et al. (2016) o interesse por temas relacionados às questões climáticos pode ser uma consequência da divulgação dos Relatórios de Avaliação do Painel Intergovernamental de Mudanças Climáticas ou Intergovernmental Panel on Climate Change (IPCC), que ressaltam os riscos do aquecimento global e instigam temas relevantes de pesquisa. Embora não inseridas na figura, foram observadas cinco publicações pontuais distribuídas nos anos 1972, 1991, 1994 e 1996, sendo que duas em 1991. Esses textos merecem destaque pelo fato de que tratam diretamente de medidas de mitigação dos impactos das secas, como o caso da semeadura de nuvens para a produção de chuva, no Estado da Flórida nos Estados Unidos (SIMPSON et al., 1972). Apesar de ter provocado efeitos positivos entre os americanos, a iniciativa não logrou êxito quando experimentada no Nordeste Brasileiro (COELHO, 1985). Esse fato ilustra a necessidade de reconhecer as especificidades locais e a necessidade de realização de estudos de replicação em áreas diferentes quando se trata de estratégias dessa natureza.

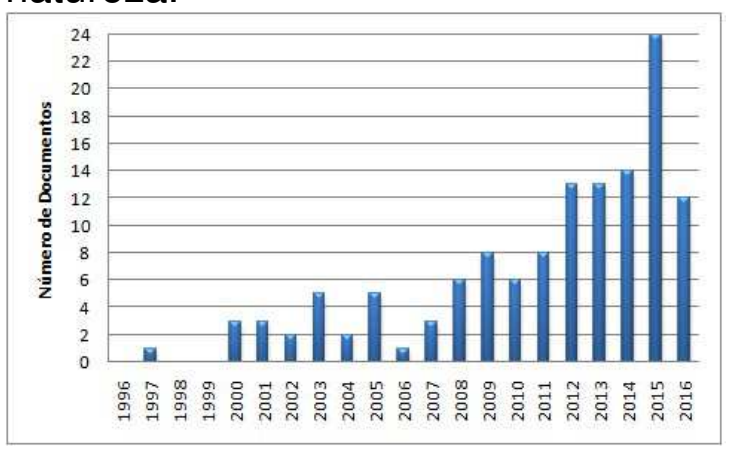

1a - Palavras de busca "mitigation" e "drought"

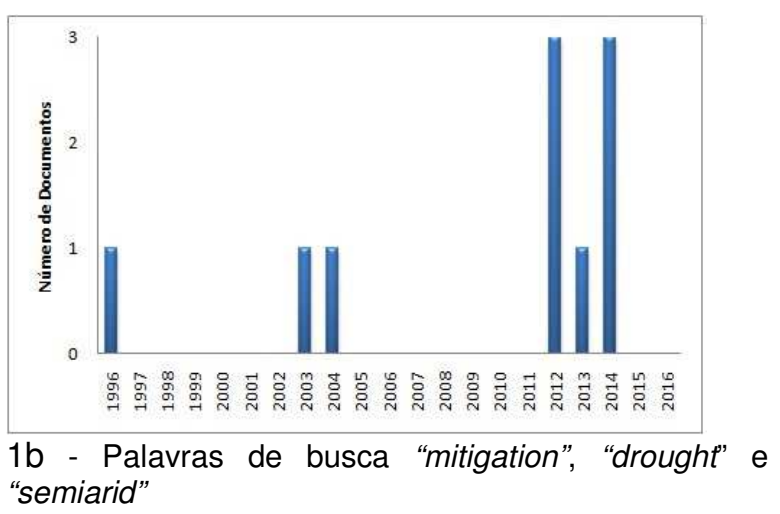

FIGURA 1 Distribuição temporal dos textos pesquisados

Fonte: site Web of Science

O artigo de Wilhite (1996) caracteriza a seca e busca diretrizes para que haja um planejamento de ações para seu enfrentamento, tanto especificamente para os Estados Unidos como para outras regiões que sofrem com as secas, caso da Ásia, África e América Latina. O artigo publicado por Lohani; Loganathan (1997) trata de uma forma amenizar os impactos da seca por meio da previsão do fenômeno a partir do Índice de Severidade de Seca de Palmer (PDSI). Os autores afirmam no texto que essa seria uma condição rápida para orientar as ações de mitigação da seca, porém estudos posteriores demonstraram que esse método não é muito eficaz (BLAIN, 2012; SIENZ et al., 2012). Outros documentos do portfólio apresentaram estudos sobre esse índice (BLAIN; MESCHIATTI, 2015; STAGGE et al., 2015). Os autores concluíram que uma adaptação do PDSI pode ser uma forma eficaz de melhorar a previsibilidade do índice. Pelo exposto, nota-se que os estudos sobre mitigação podem ser complementares e evoluir com a contribuição de outros estudiosos.

Para focar na questão da seca em regiões semiáridas, realizou-se uma nova busca no site do WoS e desta vez foram utilizadas as palavras chave drought, mitigation e semiarid. Essa nova busca apresentou um novo portfólio com 10 
trabalhos após o refinamento. O texto mais antigo é do ano de 1996, Climate change, drought and desertification (LE HOUÉROU, 1996), possuindo um número elevado de citações (486), sendo uma referência sobre o assunto. A distribuição temporal dos 10 trabalhos pode ser observada na Figura $1 \mathrm{~b}$.

Vale destacar que a distribuição dos documentos não é linear entre os anos. Percebeu-se, também, que o foco dos trabalhos voltados para as regiões semiáridas não costuma ser a mitigação da seca, mas assuntos mais abrangentes como a mudança climática.

\section{Os Tópicos mais Abordados nas Pesquisas sobre Mitigação das Secas}

Em estudos bibliométricos o mapeamento de temas dominantes, principais autores, periódicos científicos, instituições de pesquisas ou países que se destacam em um determinado domínio de conhecimento pode ser feito por meio de acoplamento bibliométrico (GRÁCIO, 2016). O acoplamento bibliográfico é um método que permite análisar semelhanças (proximidades) entre documentos a partir da análise de características comuns. Essas características podem ser palavraschave, citações, país ou instituição de origem, veículo de publicação.

Assim, a partir de um acoplamento bibliográfico no qual foram consideradas palavras-chave presentes em, no mínimo, cinco dos documentos inicialmente selecionados, foi possível identificar os temas dominantes na pesquisa sobre mitigação das secas. A hipótese adotada no acoplamento segue os moldes de Kessler (1963) criador do método, ou seja, dois documentos são considerados próximos do ponto de vista temático, se tem em comum a mesma palavra-chave. Quanto maior o número de palavras-chave compartilhadas, maior a proximidade entre os documentos. Além disso, quanto maior a quantidade de documentos com a mesma palavra-chave, maior a representatividade dessa palavra-chave como tópico de estudo.

A Figura 2 foi elaborada com o auxílio do software VOSviewer e apresenta o mapeamento dos termos mais comuns na literatura pesquisada. O VOSviewer agrupa as palavras-chave com base na co-ocorrência ou presença em diferentes documentos, e as posiciona em grupos (clusters) de acordo com a distância entre estas. A distância entre as palavras-chave é proporcional à similaridade entre elas quanto às características dos documentos em comum, por exemplo, palavras citadas em documentos com referências semelhantes, são dispostas mais próximas umas das outras do que palavras citadas em documentos que apresentam referências não coincidentes. Os círculos representam as palavras-chave mais frequentes no portfólio de 134 textos selecionados. Círculos da mesma cor fazem parte de um mesmo agrupamento. O tamanho do círculo é proporcional ao número de textos encontrados com a palavra-chave.

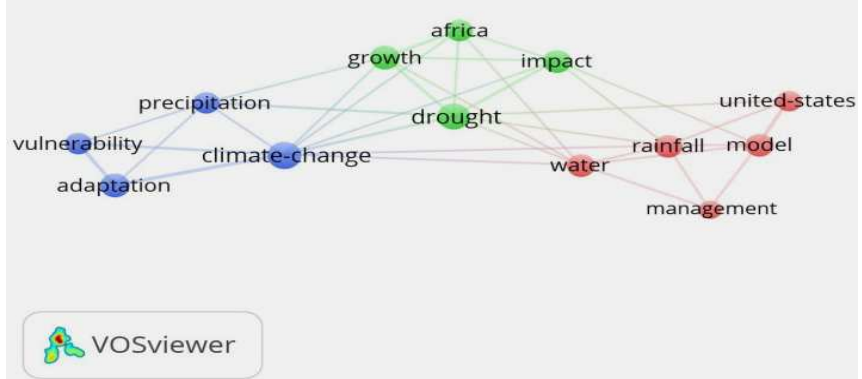

FIGURA 2 Mapa de co-ocorrência das palavras-chave no portfólio de documentos sobre mitigação das secas

Fonte: Elaboração própria com auxílio do software VOSviewer 
Das 13 palavras-chave selecionadas aquelas com maior ocorrência foram climate-change, drought, United State, water e management. Três tópicos principais agrupam conjuntos de palavras que têm maior similaridade entre si a partir da força de ligação. Esses tópicos sintetizam a tendência das pesquisas sobre mitigação das secas no período de estudo. O primeiro, formado pelos círculos azuis, agrupa trabalhos relacionados às mudanças climáticas sendo a preocupação as questões voltadas para a vulnerabilidade da população e a criação de capacidade adaptativa. O grupo verde, posicionado no centro da figura, enfatiza temas mais diretamente relacionados as secas e seus impactos. Nesse grupo nota-se a preocupação dos pesquisadores com a problemática da escassez de chuvas no continente africano. $O$ terceiro grupo, em vermelho, reúne trabalhos de natureza essencialmente teórica cujo foco volta-se para a gestão das secas e modelagem climática, sendo perceptível a importância dos pesquisadores dos Estados Unidos nesse tópico.

A palavra drought não é a mais citada entre as palavras-chave. No entanto, é tema central e co-ocorre com todas as demais palavras-chave apresentadas na figura 3, demonstrando assim como esse tema possui diversas abordagens e pode ser tratado a partir de uma análise multidisciplinar. Ainda merece destaque na figura anterior, o aparecimento do nome de dois países: África e Estados Unidos. Podendo-se inferir que dentre os trabalhos estudados, esses são os países para os quais estão se dirigindo maior atenção, com relação à temática estudada.

Os tópicos abordados nos documentos sobre mitigação das secas em regiões semiáridas são relativamente mais diversificados, como observado na Figura 3. Três regiões distintas são estudadas nos documentos, cada uma com interesses diferentes. No Brasil, grupo azul, as palavras-chave mais frequentes foram inundações e tendências observando-se o interesse por questões hídricas. Na Ásia, grupo verde, palavras como fluxos de calor, evapotranspiração e temperatura, caracterizam uma preocupação com a elevação da temperatura terrestre.

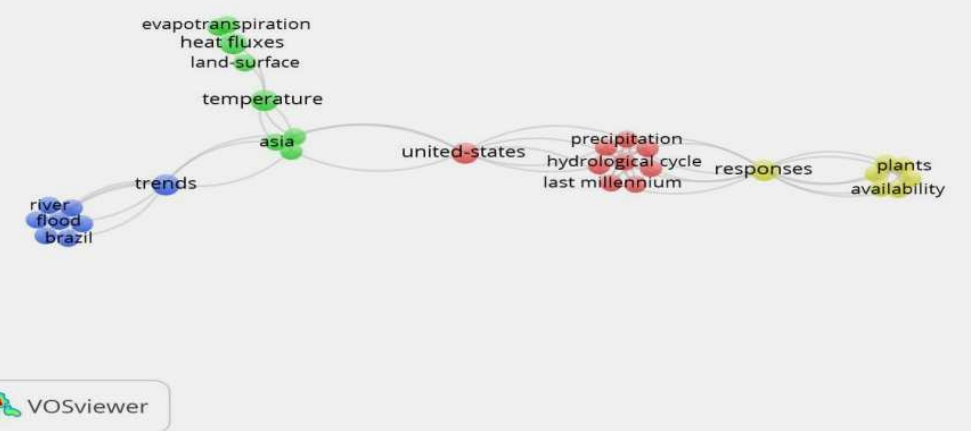

FIGURA 3 Mapa de co-ocorrência das palavras-chave no portfólio de documentos sobre mitigação das secas em regiões semiáridas

Fonte: Elaboração própria com auxílio do software VOSviewer

Nos círculos vermelhos, grupo no qual se insere os Estados Unidos, destacam-se questões relacionadas aos ciclos hidrológicos. No grupo verde apresenta-se a palavra chave Ásia e, junto as palavras como eventos climáticos extremos, fluxo de calor, caracterizando as pesquisas deste grupo como mais direcionadas às questões de mudança climática. No grupo com círculos amarelos constam estudos que buscam entender a resposta da vegetação aos impactos da seca. 


\section{A Distribuição Espacial das Pesquisas}

As secas se distribuem espacialmente em todo o mundo, não estando confinadas a uma localidade. Assim, é natural o interesse de pesquisadores de diferentes países. Tal interesse internacional também se reveste de importância à medida que o entendimento de especificidades regionais e a produção de conhecimento local sobre mitigação dos impactos da seca pode fundamentar estudos nas mais diversas áreas afetadas pelo fenômeno.

O estudo bibliométrico mostrou que 15 países se destacam na autoria de documentos sobre mitigação (Tabela 1). Das 134 publicações selecionadas, 45,52\% partiram dos Estados Unidos, China e Índia. Boa parte dos países destacados sofre com secas recorrentes e inundações. O Brasil, apesar da gravidade e do impacto que as secas causam no bem estar de milhões de famílias brasileiras, não figura entre as principais origens de pesquisas na temática, quando a análise se refere a estudos divulgados para a comunidade internacional via WoS.

TABELA 1. Distribuição espacial das publicações envolvendo o tema mitigação da seca. Período 1970-2016.

\begin{tabular}{ccc}
\hline Países & Número de publicações & Proporção \\
\hline Estados Unidos & 35 & $26,12 \%$ \\
China & 16 & $11,94 \%$ \\
Índia & 10 & $7,46 \%$ \\
Itália & 9 & $6,72 \%$ \\
Austrália & 8 & $5,97 \%$ \\
Alemanha & 8 & $5,97 \%$ \\
Inglaterra & 7 & $5,22 \%$ \\
França & 5 & $3,73 \%$ \\
Países Baixos & 5 & $3,73 \%$ \\
África do Sul & 5 & $3,73 \%$ \\
Japão & 4 & $2,98 \%$ \\
México & 4 & $2,98 \%$ \\
Bélgica & 3 & $2,24 \%$ \\
Brasil & 3 & $2,24 \%$ \\
Bulgária & 3 & $2,24 \%$ \\
\hline Total & $\mathbf{1 3 4}$ & $\mathbf{1 0 0} \%$ \\
\hline
\end{tabular}

Fonte: Elaboração própria de acordo com dados obtidos no site Web of Science

\section{Os Principais Pesquisadores e as Redes de Cooperação - Tendências na Pesquisa Colaborativa}

A Figura 4 fornece o mapa dos autores com mais publicações entre os documentos selecionados e as redes ou conexões entre esses autores. Com relação à autoria dos 134 documentos, há 431 autores. Porém, a rede de relacionamento entre estes é muito fraca o que dá origem a um mapa com muitos autores dispersos. Assim, para destacar os autores com maior número de documentos optou-se por mostrar apenas aqueles que possuem no mínimo duas publicações, o que corresponde a um grupo de 18 autores. 


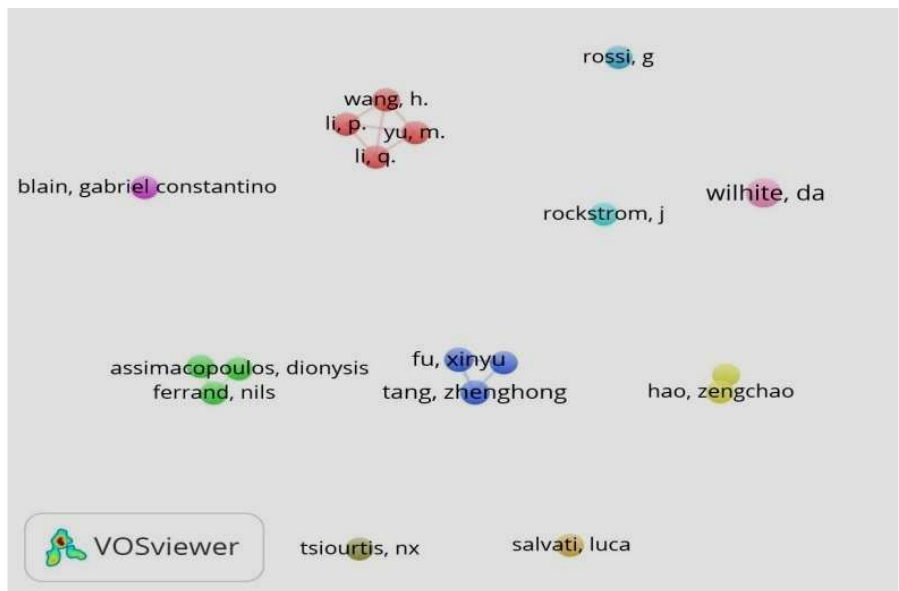

FIGURA 4 Mapa da rede de autores que possuem maior número de publicações

Fonte: Elaboração própria com auxílio do software VOSviewer

Zhao; Strotmann (2008) aplicaram o acoplamento bibliográfico de autores para mapear a estrutura de influência entre pesquisadores e as redes de pesquisa. No caso das pesquisas sobre mitigação das secas observou-se uma baixa conexão entre os estudiosos do tema. A integração entre os pesquisadores de mitigação das secas é muito baixa, sendo identificados vários clusters e poucas ligações. Não existe uma rede densa em que há vários relacionamentos, apenas pequenas redes, como a que está representada pelos círculos azuis, que são de autores da Universidade de Nebraska dos Estados Unidos, e o grupo em vermelho representado por pesquisadores de instituições chinesas.

$\mathrm{O}$ autor com maior número de documentos (Wilhite), indicado pelo maior círculo maior, não possui ligação com nenhum outro autor. Vale ressaltar que dois artigos, de origem norte americana, tratavam de questões da seca no Brasil e que tinham como autor comum aos dois textos a brasileira Maria Carmem Lemos, que é professora da School of Natural Resources and Environment, na Universidade Michigan. Outro destaque vai para o autor Blain, que escreveu dois dos três artigos brasileiros.

$\mathrm{Na}$ Figura 5 pode-se observar a rede de relacionamentos dos 18 autores segundo os seus países, ficando explicitadas as relações que existem entre China com Canadá, Japão e Austrália. Os Estados Unidos, apesar de ser o país com maior número de publicações, não apresenta conexão relevante com pesquisadores de outros países. O Brasil não aparece nessa figura por possuir apenas um documento e ter se relacionado apenas com autores dos Estados Unidos. Esse documento foi oriundo da Universidade Federal do Ceará em parceria com o Instituto Tecnológico de Massachusetts e tinha como objeto de estudos a rede de reservatórios da bacia do alto Jaguaribe que se localiza no estado do Ceará, para implementar um modelo de hidrológico de fluxo de água como o objetivo de haver um armazenamento de água mais eficaz e assim mitigar as secas (MAMEDE et al., 2012). Esse foi o único estudo realizado no Brasil, mas é referência para a ciência, pois já foi citado por 17 outros autores, demonstrando a presença do Brasil na comunidade científica internacional com relação ao tema estudado. 


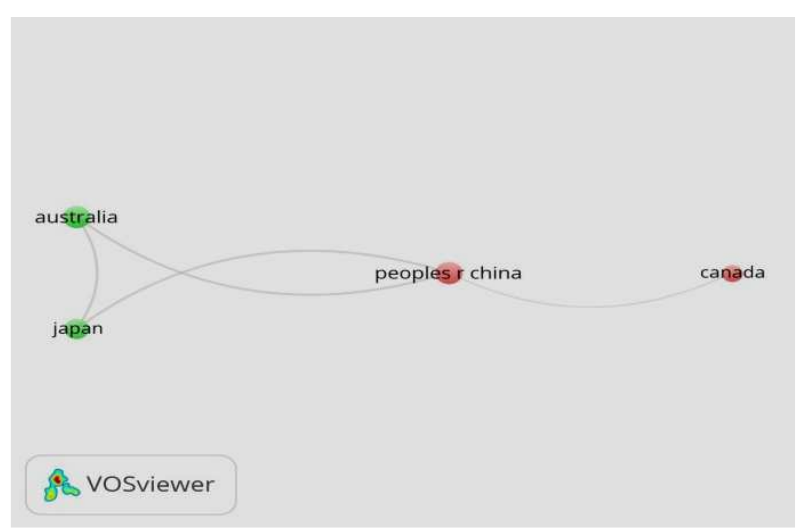

FIGURA 5. Mapa da rede de autores por países de publicação dos documentos

Fonte: Elaboração própria com auxílio do software VOSviewer

O Quadro 2 apresenta informações sobre os documentos envolvendo os termos drought, mitigation e semiarid. Apesar do número reduzido, pode-se observar que são estudos respaldados pelo fato de que, a maioria, possui muitas citações, ou seja, são trabalhos reconhecidos pela comunidade científica (MACIAS-CHAPULA, 1998).

QUADRO 2 Portfólio de artigos selecionados a partir das palavras drought, mitigation e semiarid

\begin{tabular}{|c|c|c|}
\hline Autores & Título do artigo & $\begin{array}{l}\text { Número } \\
\text { de } \\
\text { citações }\end{array}$ \\
\hline LeHouerou, H. N. & Climate change, drought and desertification & 202 \\
\hline Morton, J.; Barton, D. & $\begin{array}{l}\text { Destocking as a drought-mitigation strategy: Clarifying } \\
\text { rationales and answering critiques }\end{array}$ & 14 \\
\hline $\begin{array}{l}\text { Caravaca, F.; Alguacil, M. D.; } \\
\text { Diaz, G.; Roldan, A. }\end{array}$ & $\begin{array}{l}\text { Use of nitrate reductase activity for assessing } \\
\text { effectiveness of mycorrhizal symbiosis in } \\
\text { Dorycniumpentaphyllum under induced water deficit }\end{array}$ & 17 \\
\hline $\begin{array}{l}\text { Mamede, G. L.; Araujo, N. A. } \\
\text { M.; Schneider, C. M.; de. } \\
\text { Araujo, J. C.; Herrmann, H. J. }\end{array}$ & Overspill avalanching in a dense reservoir network & 26 \\
\hline $\begin{array}{l}\text { Ma, W.;Hafeez, M.; Rabbani, } \\
\text { U.; Ishikawa, H.; Ma, Y. }\end{array}$ & $\begin{array}{l}\text { Retrieved actual ET using SEBS model from Landsat- } 5 \\
\text { TM data for irrigation area of Australia }\end{array}$ & 18 \\
\hline $\begin{array}{l}\text { Sheng, M.; Hamel, C.; } \\
\text { Fernandez, M. R. }\end{array}$ & $\begin{array}{l}\text { Cropping practices modulate the impact of glyphosate on } \\
\text { arbuscularmycorrhizal fungi and rhizosphere bacteria in } \\
\text { agroecosystems of the semiarid prairie }\end{array}$ & 6 \\
\hline $\begin{array}{c}\text { Ma, W.; Hafeez, M.; Ishikawa, } \\
\text { H.; Ma, Y. }\end{array}$ & $\begin{array}{l}\text { Evaluation of SEBS for estimation of actual } \\
\text { evapotranspiration using ASTER satellite data for } \\
\text { irrigation areas of Australia }\end{array}$ & 8 \\
\hline $\begin{array}{l}\text { Abbas, F.; Ahmad, A.; Safeeq, } \\
\text { M.; Ali, S.; Saleem, F.; } \\
\text { Hammad, H. M.; Farhad, W. }\end{array}$ & $\begin{array}{l}\text { Changes in precipitation extremes over arid to semiarid } \\
\text { and subhumid Punjab, Pakistan }\end{array}$ & 4 \\
\hline Manalil, S.; Flower, K. & $\begin{array}{l}\text { Soil water conservation and nitrous oxide emissions from } \\
\text { different crop sequences and fallow under } \\
\text { Mediterranean conditions }\end{array}$ & 0 \\
\hline $\begin{array}{l}\text { Ault, T. R.; Cole, J. E.; } \\
\text { Overpeck, J. T.; Pederson, G. } \\
\text { T.; Meko, D. M. }\end{array}$ & $\begin{array}{l}\text { Assessing the Risk of Persistent Drought Using Climate } \\
\text { Model Simulations and Paleoclimate Data }\end{array}$ & 30 \\
\hline
\end{tabular}

Fonte: Elaboração própria de acordo com dados obtidos no site Web of Science

ENCICLOPÉDIA BIOSFERA, Centro Científico Conhecer - Goiânia, v.14 n.26; p.1248 


\section{CONCLUSÕES}

A análise da produção do conhecimento sobre "mitigação das secas" permitiu identificar três abordagens distintas entre os documentos pesquisados: i) a abordagem da mitigação na perspectiva das mudanças climáticas, enfatizando a vulnerabilidade e a capacidade de adaptação da população, ii) a abordagem mais empírica, voltada para os impactos socioeconômicos das secas (nesse grupo de documentos percebe-se a sensibilidade dos pesquisadores às condições enfrentadas pelos países africanos) e iii) a abordagem mais teórica, que prioriza a modelagem climática. Em se tratando de mitigação há escassez de estudos voltados para questões envolvendo governança, avaliação de políticas públicas para enfrentamento das secas e participação da população em processos decisórios.

Além disso, foram encontrados poucos estudos voltados para as regiões semiáridas, apesar das projeções de intensificação das secas nessas áreas nos próximos anos. Esses aspectos certamente podem orientar pesquisas futuras.

Além da proposição de novos tópicos de pesquisa o artigo contribuiu para a identificação dos autores mais produtivos e para ressaltar a baixa conexão entre aqueles que estudam a mitigação das secas. Essa falta de integração ou colaboração científica entre os pesquisadores pode ser um fator inibidor de avanços qualitativos nessa temática seja em escala global ou regional.

Como a maioria das análises bibliométricas, este estudo tem algumas limitações que o tornam de natureza preliminar e exploratória: i) a impossibilidade de adoção de uma base de dados de abrangência global, capaz de incorporar todos os documentos publicados na temática do estudo, ii) a impossibilidade de aprofundar a discussão de todos os assuntos levantados nos documentos selecionados, mais especificamente dos impactos provocados pelas pesquisas e a iii) predominância de indicadores quantitativos que impedem análises como a contribuição dos países e autores mais produtivos para a minimização dos impactos das secas. Assim, como sugestão de novos estudos, propõe-se uma análise bibliométrica com a inclusão de documentos de bases nacionais e internacionais, a inclusão de outros indicadores bibliométricos como o número de co-citações, bem como análises pontuais sobre os desdobramentos e impactos da produção científica sobre mitigação das secas.

\section{REFERÊNCIAS}

BLAIN, G. C. Revisiting the probabilistic definition of drought: strengths, limitations and an agrometeorological adaptation. Bragantia, v. 71, n. 1, p. 132-141, 2012. Disponível em: < http://dx.doi.org/10.1590/S0006-87052012000100019>. Doi: $10.1590 /$ S0006-87052012000100019

BLAIN, G. C.; MESCHIATTI, M. C. Inadequacy of the gamma distribution to calculate the Standardized Precipitation Index. Revista Brasileira de Engenharia Agrícola e Ambiental, v. 19, n. 12, p. 1129-1135, 2015 . Disponível em:<http://dx.doi.org/10.1590/1807-1929/agriambi.v19n12p1129-1135>.Doi: 10.1590/1807-1929/agriambi.v19n12p1129-1135

BORNMANN, L.; MUTZ, R. Growth rates of modern science: A bibliometric analysis based on the number of publications and cited references. Journal of the Association for Information Science and Technology, v. 66, n. 11, p. 2215-2222, 2015. Disponível em: <https://doi.org/10.1002/asi.23329>. doi: 10.1002/asi.23329 
COELHO, J. As secas do Nordeste e a indústria das secas. Petrópolis: Vozes, 1985.

COLEPÍCOLO, E. Análise bibliométrica sobre a produção científica no campo das habilidades sociais. Biblios: Revista electrónica de bibliotecología, archivología y museología, n. 57, p. 4, $2014 . \quad$ Disponível em:<https://doi.org/10.5195/biblios.2014.191>doi: 10.5195/biblios.2014.191

DZIEGIELEWSKI, B. Long-term and short-term measures for coping with drought. In: ROSSI, G. (Coordenador), Tools for drought mitigation in Mediterranean Regions. Springer Science \& Business Media, 2003. p. 319-339. Disponível em: <https://doi.org/10.1007/978-94-010-0129-8_19 >. Doi: 10.1007/978-94-010-01298_19

ENSSLIN, L.; ENSSLIN, S. R.; PACHECO, G. C. Um estudo sobre segurança em estádios de futebol baseado na análise bibliométrica da literatura internacional. Perspectivas em Ciência da Informação, v.17, n.2, p.71-91, 2012. Disponível em: <https://doi.org/10.1590/s1413-99362012000200006>. doi: 10.1590/s141399362012000200006

GRÁCIO, M. C. C. Acoplamento bibliográfico e análise de cocitação: revisão teóricoconceitual. Encontros Bibli: Revista Eletrônica de Biblioteconomia e Ciência da Informação, v. $21, \quad$ n. $47, \quad$ p. 82-99, $2016 . \quad$ Disponível em:<http://dx.doi.org/10.5007/1518-2924.2016v21n47p82>. doi: 10.5007/1518-2924.

HAUNSCHILD, R.; BORNMANN, L.; MARX, W. Climate Change Research in View of Bibliometrics. PLoS ONE, v.11, n. 7, e0160393, 2016. Disponível em:<https://doi.org/10.1371/journal.pone.0160393>. doi: 10.1371/journal.pone.0160393.

IPCC - Intergovernmental Panel on Climate Change.Climate Change 2014. Climate Change 2014: Impacts, Adaptation, and Vulnerability. Part B: Regional Aspects.Contribution of Working Group II to the Fifth Assessment Report of the Intergovernmental Panel on Climate Change. Cambridge University Press, Cambridge, United Kingdom and New York, NY, USA.pp. 1499 -1566. 2014.

JI, Q.; PANG, X.; ZHAO, X.A bibliometric analysis of research on Antarctica during 1993-2012. Scientometrics, v.101, n.3, p. 1925-1939, 2014. Disponível em: <https://doi.org/10.1007/s11192-014-1332-5>. doi: 10.1007/s11192-014-1332-5

KESSLER, M. M. Bibliographic coupling between scientific papers. American Documentation, v. 14, n. 1, p. 10-25, 1963. Disponível em: <https://doi.org/10.1002/asi.5090140103>. Acesso em 24 de novembro de 2016.doi: 10.1002/asi.5090140103.

LE HOUÉROU, H. N. Climate change, drought and desertification. Journal of Arid Environments, v. 34, n. 2, p. 133-185, 1996. Disponível em:<https://doi.org/10.1006/jare.1996.0099>. doi: 10.1006/jare.1996.0099

LOHANI, V. K.; LOGANATHAN, G. V.An early warning system for drought ENCICLOPÉDIA BIOSFERA, Centro Científico Conhecer - Goiânia, v.14 n.26; p.1250 
management using the Palmer drought index. Journal of the American Water Resources Association, v. 33, n.6, p. 1375-1386, 1997. Disponível em:<https://doi.org/10.1111/j.1752-1688.1997.tb03560.x>. doi: 10.1111/j.1752-1688.

MACIAS-CHAPULA, C. A. O papel da informetria e da cienciometria e sua perspectiva nacional e internacional. Ciência da Informação, v. 27, n. 2, p. 134-140, 1998. Disponível em: <https://doi.org/10.1590/s0100-19651998000200005 >. Acesso em: 13 dezembro. 2016. Doi: 10.1590/s0100-19651998000200005.

MAMEDE, G. L.; ARAÚJO, N. A. M.; SCHNEIDER, C. M.; ARAÚJO, J. C.; HERRMANN, H. J. Overspill avalanching in a dense reservoir network. Proceedings of the National Academy of Sciences, v. 109, n.19, p. 7191-7195, 2012. Disponível em:<https://doi.org/10.2139/ssrn.2191634>. Doi: 10.2139/ssrn.2191634.

MANCAL, A.; LIMA, P. V. P. S.; KHAN, A. S.; MAYORGA, M. I. O. À espera da seca que vem: capacidade adaptativa em comunidades rurais do semiárido. Revista Brasileira de Estudos de População, v.33, n.2, p. 257-281, 2016. Disponível em: <https://doi.org/10.20947/s0102-30982016a0012> . doi: 10.20947/s0102$30982016 \mathrm{a} 0012$

MARX, W.;HAUNSCHILD, R.; THOR, A.; BORNMANN, L. Which early works are cited most frequently in climate change research literature? A bibliometric approach based on Reference Publication Year Spectroscopy. Scientometrics, p. 1-19, 2016. Disponível em: <https://doi.org/10.1007/s11192-016-2177-x> . doi: 10.1007/s11192016-2177-x

MIYAN, M. A. Droughts in Asian least developed countries: Vulnerability and sustainability. Weather and Climate Extremes, v. 7, p. 8-23, 2015. Disponível em:<http://dx.doi.org/10.1016/j.wace.2014.06.003>.

Doi: 10.1016/j.wace.2014.06.003.

ROSSI, G.; CANCELLIERE, A. At-site and regional drought identification by REDIM model.In: ROSSI, Giuseppe. (Coordenador), Tools for drought mitigation in Mediterranean Regions. Springer Science \& Business Media, 2003. p.37-54.. Disponível em: <https://doi.org/10.1007/978-94-010-0129-8_3>. Doi: 10.1007/97894-010-0129-8_3.

SIENZ, F.; BOTHE, O.; FRAEDRICH, K. Monitoring and quantifying future climate projections of dryness and wetness extremes: SPI bias. Hydrology and Earth System Sciences, v. 16, n. 7, p. 2143, 2012. Disponível em: <https://doi.org/10.5194/hessd-8-10635-2011>. doi:10.5194/hess-16-2143-2012.

SILVEIRA, J. P. B. A produção científica em periódicos institucionais: um estudo da revista biblos. Revista Eletrônica de Biblioteconomia e Ciência da Informação, v. 17, n. 33, p. 116-133, 2012. Disponível em: <https://doi.org/10.5007/15182924.2011v17n33p116>. Doi: doi.org/10.5007/1518-2924

SIMPSON, J.; WOODLEY, W. L.; WHITE, R. M. Joint Federal-State Cumulus Seeding Program for Mitigation of South Florida Drought. Bulletin of the American 
Meteorological Society, v. 53, n.4, p. 334-344, 1972. Disponível em: < https://doi.org/10.1175/1520-0477(1972)053\%3C0334:jfscsp\%3E2.0.co;2>. Doi: 10.1175/1520-0477

STAGGE, J. H.; TALLAKSEN, L. M.; GUDMUNDSSON, L.; VAN LOON, A. F.; STAHL, K. Candidate distributions for climatological drought indices (SPI and SPEI).International Journal of Climatology, v. 35, n. 13, p. 4027-4040, 2015. Disponível em: <https://doi.org/10.1002/joc.4267>. doi: 10.1002/joc.4267

VAN LOON, A. F. Hydrological drought explained. Wiley Interdisciplinary Reviews: Water, v. 2, n. 4, p. 359-392, 2015. Disponível em: <https://doi.org/10.1002/wat2.1085>.Doi: 10.1002/wat2.1085

WANG, B.; PAN, S. Y.; KE, R. Y.; WANG, K.; WEI, Y. M.; An overview of climate change vulnerability: a bibliometric analysis based on Web of Science database. Natural Hazards, v. 74, n. 3, p. 1649-1666, 2014. Disponível em: <https://doi.org/10.1007/s11069-014-1260-y>. doi: 10.1007/s11069-014-1260-y

WILHITE, D. A. A Methodology for Drought Preparedness. Natural Hazards, v. 13, n.3, p. 229-252, 1996. Disponível em: < https://doi.org/10.1007/bf00215817>. Doi: $10.1007 /$ bf00215817

ZHAO, D.; STROTMANN, A. Evolution of research activities and intellectual influences in information science 1996-2005: Introducing author bibliographic-coupling analysis. Journal of the American Society for Information, v. 59, n. 13, p. 2070-2086, 2008. Disponível em: <https://doi.org/10.1002/asi.20910>. Doi: $10.1002 /$ asi.20910 